\title{
Atom-mediated spontaneous parametric down-conversion in periodic waveguides
}

\author{
Sina Saravi ${ }^{1,2,}$, Alexander N. Poddubny $2,3,4$, Thomas Pertsch ${ }^{1}$, Frank Setzpfandt ${ }^{1}$, And \\ ANDREY A. SUKHORUKOV ${ }^{2}$ \\ ${ }^{1}$ Institute of Applied Physics, Abbe Center of Photonics, Friedrich Schiller University Jena, Albert-Einstein-Strasse 6, 07745 Jena, Germany \\ ${ }^{2}$ Nonlinear Physics Centre, Research School of Physics and Engineering, Australian National University, Canberra, ACT 2601, Australia \\ ${ }^{3}$ ITMO University, St. Petersburg 197101, Russia \\ ${ }^{4}$ loffe Institute, St. Petersburg 194021, Russia \\ *Corresponding author: sina.saravi@uni-jena.de \\ Compiled October 16, 2017
}

We propose the concept of atom-mediated spontaneous parametric down-conversion (SPDC), where photonpair generation can only take place in the presence of a single 2-level emitter, relying on the bandgap evanescent modes of a nonlinear periodic waveguide. Using a guided signal mode, an evanescent idler mode, and an atom-like emitter with idler's transition frequency embedded in the structure, we find a heralded excitation mechanism, where the detection of a signal photon outside the structure heralds the excitation of the embedded emitter. We use a rigorous Green's function quantization method to model this heralding mechanism in a 1D periodic waveguide and determine its robustness against losses. () 2017 Optical Society of America

OCIS codes: (190.4410) Nonlinear optics, parametric processes; (350.4238) Nanophotonics and photonic crystals; (270.4180) Quantum optics, Multiphoton processes; (020.1335) Atom optics.

http://dx.doi.org/10.1364/ao.XX.XXXXXX

Photonic crystals (PCs) are structures with a periodically varying permittivity. They offer strong control of light-matter interactions on the subwavelength scale [1] and are a valuable resource in integrated and large-scale implementations of quantum optical technologies [2]. The dispersion properties of Bloch modes in nonlinear PCs can be designed to enhance [3] and engineer [4] sources of photon-pairs. Furthermore, PCs enable engineering of the emission and interaction of single-emitters, e.g. cold atoms [5-7] or solid-state emitters [8-11].

In most applications with an emitter, PCs are used to alter the density of states (DOS), and thereby enhance or inhibit the emission [9]. However, when the emitter's transition frequency is within the bandgap of the PC, where evanescent modes have zero DOS [12], it is the emitter that alters the PC, making the DOS non-zero by adding a free state to the system. Such a state is referred to as an atom-photon bound state [13] or an atominduced cavity [6], which allows engineering of atom-atom and atom-photon interactions [6, 7].

In this Letter, we suggest new possibilities in tailoring quan-

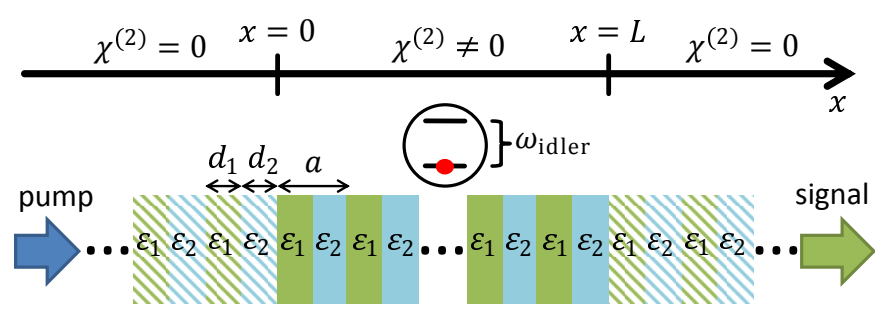

Fig. 1. Schematic of atom-mediated SPDC.

tum light-matter interactions through a combination of nonlinear parametric processes and atom-induced states. Specifically, we consider SPDC, a process widely used to generate photonpairs for quantum optical applications [14], in which a pump photon splits into a pair of entangled lower frequency photons, called signal and idler. SPDC sources can produce photon-pairs of widely tunable frequencies and degrees of entanglement [15], although multi-pair emission limits their purity [16]. We find that a single emitter placed in a nonlinear PC can mediate SPDC, where parametric generation of one photon becomes conditional on the absorption of a paired photon by the emitter, while simultaneous generation of extra pairs gets suppressed.

To investigate atom-mediated SPDC, we consider a simplified model: an infinite 1D periodic dielectric stack, shown in Fig. 1, where the $\chi^{(2)}$ nonlinearity only exists within $0<x<L, x$ being the propagation direction along the periodicity of the structure. The phase-matching condition is set to be satisfied between a propagating pump, a propagating signal, and a bandgap idler mode. We show that the zero DOS of the evanescent idler mode completely prohibits SPDC, yet it can be mediated by a single 2-level emitter with the idler's transition frequency, placed near or inside the nonlinear region, providing a single state for the idler's quantum of excitation. Then, the detection of a signal photon would herald the emitter's excitation.

We aim to describe SPDC involving evanescent modes and also account for possible losses. Since under such conditions the modes are non-power-orthogonal, one cannot expand the state of the system into photons as quanta of energy associated 
to a certain mode [17]. Consequently, throughout this work, we use the term "photon" loosely, to refer to quanta of local excitation in the medium-assisted field with the local bosonic annihilation operator $\hat{f}(\boldsymbol{r}, \omega)$, satisfying the commutation relation $\left[\hat{f}(\boldsymbol{r}, \omega), \hat{f}^{\dagger}\left(\boldsymbol{r}^{\prime}, \omega^{\prime}\right)\right]=\delta\left(\boldsymbol{r}-\boldsymbol{r}^{\prime}\right) \delta\left(\omega-\omega^{\prime}\right)[18]$. To simplify notations, we assume that the only non-zero element of the quadratic nonlinear tensor is $\chi_{z z z}^{(2)}=\chi^{(2)}$ and the dipole moments for the atoms are oriented along $z$. Consequently, in the 1D system only the $z$-components of vectors participate in the interactions, creating a scalar scenario. We note that our approach can also account for vectorial effects if required.

We use a recently developed theoretical framework for analysing SPDC in the presence of 2-level emitters [19] based on the Green's function (GF) quantization method [18], that is capable of taking into account evanescent modes of the system. To investigate SPDC with and without the mediation of the idler atom, we formally introduce a signal atom to model a single-photon detector. Then, we compare two quantities: $P_{\text {is }}\left(\boldsymbol{r}_{s}, \boldsymbol{r}_{i}, \omega_{s}, \omega_{i}\right)$ the probability of two atoms of transition frequencies $\omega_{s}$ and $\omega_{i}$ (we refer to them as signal and idler atoms) placed at $\boldsymbol{r}_{S}$ and $\boldsymbol{r}_{i}$ both being excited through SPDC with no excitation remaining in the medium-assisted field, and $P_{S}\left(\boldsymbol{r}_{S}, \omega_{S}\right)$ the probability of the signal atom at position $\boldsymbol{r}_{s}$ being excited through SPDC with the idler photon remaining in the field and not being absorbed by the idler atom.

The probability $P_{s}=\int d^{3} \boldsymbol{r}_{i} \int_{0}^{\infty} d \omega_{i}\left|\left\langle f\left|V^{(2)}\right| i\right\rangle\right|^{2}$, accounting for all the spatial and spectral possibilities for the generated idler photon, is calculated using second-order perturbation theory. Here $V^{(2)}=\left(\frac{-i}{\hbar}\right)^{2} \int_{-\infty}^{+\infty} d t_{1} \int_{-\infty}^{t_{1}} d t_{2} \hat{H}_{\text {int }}\left(t_{1}\right) \hat{H}_{\text {int }}\left(t_{2}\right)$, with interaction Hamiltonian $\hat{H}_{\text {int }}=\hat{H}_{\mathrm{NL}}+\hat{H}_{s}$, initial vacuum state $|i\rangle=|0\rangle$, and final state $|f\rangle=\hat{f}^{\dagger}\left(\boldsymbol{r}_{i}, \omega_{i}\right) \hat{a}_{s}^{\dagger}|0\rangle$. The probability $P_{\text {is }}=\left|\left\langle f\left|V^{(3)}\right| i\right\rangle\right|^{2}$ is calculated using the third-order perturbation theory, where $V^{(3)}=$ $\left(\frac{-i}{\hbar}\right)^{3} \int_{-\infty}^{+\infty} d t_{1} \int_{-\infty}^{t_{1}} d t_{2} \int_{-\infty}^{t_{2}} d t_{3} \hat{H}_{\text {int }}\left(t_{1}\right) \hat{H}_{\text {int }}\left(t_{2}\right) \hat{H}_{\text {int }}\left(t_{3}\right), \hat{H}_{\text {int }}=$ $\hat{H}_{\mathrm{NL}}+\hat{H}_{i}+\hat{H}_{s},|i\rangle=|0\rangle$, and $|f\rangle=\hat{a}_{i}^{\dagger} \hat{a}_{s}^{\dagger}|0\rangle . \hat{H}_{\mathrm{NL}}(t) \propto$ $\varepsilon_{0} \int d^{3} \boldsymbol{r} \chi^{(2)}(\boldsymbol{r}) E_{p}(\boldsymbol{r}, t) \hat{E}(\boldsymbol{r}, t) \hat{E}(\boldsymbol{r}, t)$ is the semi-classical nonlinear Hamiltonian for the pair generation process in the interaction picture. The classical pump pulse $E_{p}(\boldsymbol{r}, t)=$ $\int_{-\infty}^{+\infty} d \omega_{p} E_{p}\left(\boldsymbol{r}, \omega_{p}\right) e^{-i \omega_{p} t}$ is treated in the undepleted pump approximation assuming a weak interaction. The electric field operator in the interaction picture is $\hat{E}(\boldsymbol{r}, t)=\int_{0}^{+\infty} d \omega \hat{E}(\boldsymbol{r}, \omega) e^{-i \omega t}+$ H.c., with $\hat{E}(\boldsymbol{r}, \omega)=i \sqrt{\frac{\hbar}{\pi \varepsilon_{0}}} \frac{\omega^{2}}{c^{2}} \int d^{3} \boldsymbol{s} \sqrt{\varepsilon^{\prime \prime}(\boldsymbol{s}, \omega)} G(\boldsymbol{r}, \boldsymbol{s}, \omega) \hat{f}(\boldsymbol{s}, \omega)$. Here, $\varepsilon^{\prime \prime}$ is the imaginary part of the relative permittivity of the material and $G$ is the Green's function satisfying $[(\nabla \times \nabla \times)-$ $\left.\frac{\omega^{2}}{c^{2}} \varepsilon(\boldsymbol{r})\right] G\left(\boldsymbol{r}, \boldsymbol{r}^{\prime}, \omega\right)=\delta\left(\boldsymbol{r}-\boldsymbol{r}^{\prime}\right) . \quad \hat{H}_{i, s}(t)=-\left(\hat{a}_{i, s} d_{i, s} e^{-i \omega_{i, s} t}+\right.$ $\left.\hat{a}_{i, s}^{\dagger} d_{i, s}^{*} e^{i \omega_{i, s} t}\right) \hat{E}\left(\boldsymbol{r}_{i, s}, t\right)$ are the dipole interaction Hamiltonians, where $a_{i, s}^{\dagger}$ are the atoms' raising operators and $d_{i, s}$ are the dipole moments. The calculation, making use of the GF identity $\operatorname{Im}\left[G\left(\boldsymbol{r}, \boldsymbol{r}^{\prime}, \omega\right)\right]=\frac{\omega^{2}}{c^{2}} \int d^{3} \boldsymbol{s} \varepsilon^{\prime \prime}(\boldsymbol{s}, \omega) G(\boldsymbol{r}, \boldsymbol{s}, \omega) G^{*}\left(\boldsymbol{r}^{\prime}, \boldsymbol{s}, \omega\right)$, results in:

$$
\begin{gathered}
P_{S} \propto\left|\frac{16 \pi d_{s}^{2}}{\hbar \varepsilon_{0}}\right| \frac{\omega_{s}^{4}}{c^{4}} \int_{0}^{\infty} d \omega_{i} \frac{\omega_{i}^{2}}{c^{2}} \int d^{3} \boldsymbol{r} \int d^{3} \boldsymbol{r}^{\prime} \Gamma\left(\boldsymbol{r}, \omega_{p}\right) \Gamma^{*}\left(\boldsymbol{r}^{\prime}, \omega_{p}\right) \\
\times \operatorname{Im}\left[G\left(\boldsymbol{r}, \boldsymbol{r}^{\prime}, \omega_{i}\right)\right] G\left(\boldsymbol{r}_{s}, \boldsymbol{r}, \omega_{s}\right) G^{*}\left(\boldsymbol{r}_{s}, \boldsymbol{r}^{\prime}, \omega_{s}\right), \\
P_{\text {is }} \propto\left|\frac{4 \pi d_{i} d_{s}}{\hbar \varepsilon_{0}}\right|^{2} \frac{\omega_{s}^{4} \omega_{i}^{4}}{c^{8}}\left|\int d^{3} \boldsymbol{r} \Gamma\left(\boldsymbol{r}, \omega_{p}\right) G\left(\boldsymbol{r}_{i}, \boldsymbol{r}, \omega_{i}\right) G\left(\boldsymbol{r}_{s}, \boldsymbol{r}, \omega_{s}\right)\right|^{2}
\end{gathered}
$$

with $\omega_{p}=\omega_{i}+\omega_{s}$ and $\Gamma\left(\boldsymbol{r}, \omega_{p}\right) \equiv \chi^{(2)}(\boldsymbol{r}) E_{p}\left(\boldsymbol{r}, \omega_{p}\right)$.

By comparing $P_{\text {is }}$ and $P_{s}$, we can determine the effect of the idler atom in the SPDC process, as in both cases the signal photon has been detected (absorbed by the signal atom), but in $P_{\mathcal{S}}$

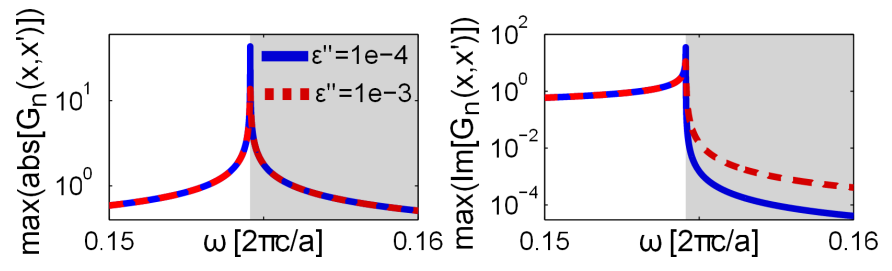

Fig. 2. The absolute value and imaginary part of the dimensionless Green's function $G_{n}\left(x, x^{\prime}, \omega\right)$, where maximum values are taken over spatial coordinates $x$ and $x^{\prime}$, for $\varepsilon^{\prime \prime}=0.001$ and $\varepsilon^{\prime \prime}=0.0001$. The gray shaded area is the bandgap region.

the idler photon remains in the field and in $P_{\text {is }}$ it excites the idler atom. We see from Eq. (1) that $P_{S}$ is exactly zero with an evanescent idler mode, as $\operatorname{Im}\left[G\left(\boldsymbol{r}, \boldsymbol{r}^{\prime}, \omega_{i}\right)\right]=0$ when $\omega_{i}$ is within the bandgap of an infinitely extended periodic structure. This means that SPDC with an idler photon remaining in the field of a purely evanescent mode is completely prohibited, as a mode with zero DOS is incapable of accepting a photon. On the other hand, according to Eq. (2), $P_{\text {is }}$ is dependent on the total GF and henceforth is not zero, as the evanescent mode still has a purely real-valued GF. In other words, the presence of the idler atom provides a free state for the quantum of excitation to be generated into, exciting the idler atom and mediating the previously prohibited SPDC process. An important implication is that in the event of a signal photon detection, we know with certainty that an idler atom has been present and excited through SPDC. This creates a heralding mechanism, where the detection of the signal photon heralds the excitation of the idler atom.

In a realistic structure however, we do not have purely evanescent modes, as loss is ever present due to either material absorption or fabrication imperfections. To quantify the heralding efficiency, we calculate the pair generation in the presence of the imaginary part $\left(\varepsilon^{\prime \prime}\right)$ of the permittivities of the layers.

Our first step is to evaluate the GF. The GF expression for a periodic structure can be found using the unconjugated reciprocity theorem $[20,21]$, so that it can include all Bloch modes, guided, evanescent, or lossy. The result is:

$$
\begin{gathered}
G\left(x, x^{\prime}, \omega\right)=\left[e^{+}(x, \omega) e^{-}\left(x^{\prime}, \omega\right) e^{i k^{+}(\omega)\left(x-x^{\prime}\right)} \Theta\left(x-x^{\prime}\right)\right. \\
\left.+e^{-}(x, \omega) e^{+}\left(x^{\prime}, \omega\right) e^{i k^{+}(\omega)\left(x^{\prime}-x\right)} \Theta\left(x^{\prime}-x\right)\right] \\
\quad \times \frac{i a c}{2 \omega} \frac{n_{g}^{+}(\omega)}{\mathcal{S} \int_{0}^{a} d x \varepsilon(x) e^{+}(x) e^{-}(x)} \equiv \frac{a}{\mathcal{S}} G_{n}\left(x, x^{\prime}, \omega\right),
\end{gathered}
$$

where we assumed a single mode present at each frequency, with $e(x)=e(x+a)$ being the Bloch mode profile, $k(\omega)$ the wavevector, and $n_{g} \equiv c / v_{g}$ the complex group-index [20] of that Bloch mode. $\Theta(x)$ is the Heaviside function. $\mathcal{S}$ is a transverse normalization area in the $y z$-plane and $G_{n}$ is a dimensionless GF normalized to $\mathcal{S}$. The + and - indices refer to forward and backward propagating modes, respectively.

We analytically find [22] the band diagram and the electricfield profiles for the 1D structure of Fig. 1 with parameters $\varepsilon_{1}=$ $3+i \varepsilon^{\prime \prime}, \varepsilon_{2}=12+i \varepsilon^{\prime \prime}$, and $d_{1}=d_{2}=0.5 a$, which we use to numerically construct $G_{n}\left(x, x^{\prime}, \omega\right)$ for different values of $\varepsilon^{\prime \prime}$. To visualize the main physics in the GF, we take the maximum of both the absolute value and the imaginary part of $G_{n}\left(x, x^{\prime}\right)$, and plot them in Fig. 2 as a function of $\omega$. We see that $\operatorname{Im}[G]$ is much smaller for the evanescent modes in the bandgap region, but it is non-zero due to the presence of a finite $\varepsilon^{\prime \prime}$. Furthermore, we see that $\operatorname{Im}[G]$ in the bandgap decreases with decreasing $\varepsilon^{\prime \prime}$. $G\left(x, x^{\prime}\right)$ becomes purely real-valued for a bandgap mode in the 
(a)

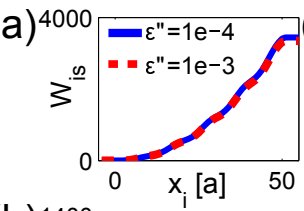

(b)

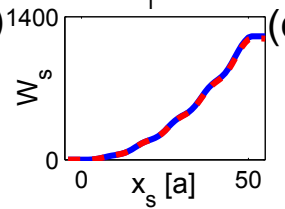

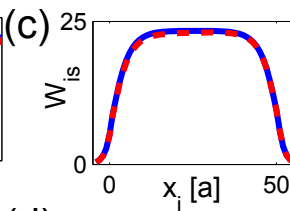

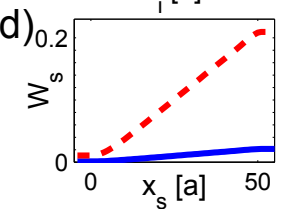

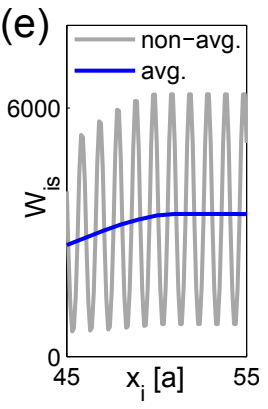

Fig. 3. The normalized pair-generation probabilities with the idler photon $(\mathrm{a}, \mathrm{c})$ absorbed by the idler atom at position $x_{i}$, $W_{\text {is }}\left(x_{s}=55 a, x_{i}\right)$, and $(\mathrm{b}, \mathrm{d})$ not absorbed by the idler atom, $W_{s}\left(x_{s}\right)$. Parameters are $(\mathrm{a}, \mathrm{b}) \omega_{s}=\omega_{i}=0.15$ (both propagating) and (c,d) $\omega_{i}=0.16$ (evanescent) and $\omega_{s}=0.15$ (propagating). Plots in (a-d) are averaged over every unit cell, and (e) shows the non-averaged (a) near the edge of the nonlinear region. $\chi^{(2)} \neq 0$ for $0<x<50 a$. Blue solid and red dashed curves correspond to $\varepsilon^{\prime \prime}=10^{-4}$ and $\varepsilon^{\prime \prime}=10^{-3}$, respectively.

lossless structure. Finally, we see that abs $[G]$ is of comparable magnitudes for guided and evanescent modes, except for the slow modes at the bandedge.

To proceed with the numerical analysis, we assume that for $\omega_{p}=\omega_{s}+\omega_{i}$, the phase-matching condition $k_{p}=\operatorname{Re}\left[k_{s}+k_{i}\right]$ is satisfied. Such modal phase-matchings for a three-wave-mixing process can be reached for realistic photonic crystal designs $[23,24]$. Then, we consider only the phase-matched pump spatial harmonic as $E_{p}\left(\boldsymbol{r}, \omega_{p}\right)=E_{p}\left(\omega_{p}\right) e^{i k_{p}\left(\omega_{p}\right) x}$. We assume a constant nonlinearity profile $\chi^{(2)}(\boldsymbol{r})=\chi^{(2)}$. We then define the normalized pair-generation probabilities $W_{\text {is }}$ and $W_{s}$, that are independent of the strength of the nonlinearity, the pump pulse's spectrum, and the atomic-dipole moments, and only include the physics associated with the photonic band gap:

$$
\begin{aligned}
& P_{\text {is }} \propto \quad\left|\frac{d_{i} d_{s} \chi^{(2)} E_{p}\left(\omega_{i}+\omega_{s}\right)}{a S \hbar \varepsilon_{0}}\right|^{2} W_{\text {is }}\left(\omega_{i}, \omega_{s}, x_{i}, x_{s}\right), \\
& W_{\mathrm{is}} \equiv 16 \pi^{2} \frac{a^{8} \omega_{s}^{4} \omega_{i}^{4}}{c^{8}}\left|\int \frac{d x}{a} e^{i k_{p} x} G_{n}\left(x_{i}, x, \omega_{i}\right) G_{n}\left(x_{s}, x, \omega_{s}\right)\right|^{2} \text {, } \\
& P_{S} \propto\left|\frac{d_{s}^{2}}{a \mathcal{S} \hbar \varepsilon_{0}}\right| \int_{0}^{\infty} d \omega_{i}\left|\chi^{(2)} E_{p}\left(\omega_{i}+\omega_{S}\right)\right|^{2} W_{\mathcal{S}}\left(\omega_{i}, \omega_{\mathcal{S}}, x_{S}\right), \\
& W_{s} \equiv \quad 16 \pi \frac{a^{6} \omega_{s}^{4} \omega_{i}^{2}}{c^{6}} \int \frac{d x}{a} \int \frac{d x^{\prime}}{a} e^{i k_{p}\left(x-x^{\prime}\right)} \\
& \times \operatorname{Im}\left[G_{n}\left(x, x^{\prime}, \omega_{i}\right)\right] G_{n}\left(x_{s}, x, \omega_{s}\right) G_{n}^{*}\left(x_{s}, x^{\prime}, \omega_{s}\right) .
\end{aligned}
$$

We use the numerically evaluated $G_{n}\left(x, x^{\prime}\right)$ in Eqs. (5) and (7) to evaluate these normalized pair-generation probabilities. The results are plotted in Fig. 3 as a function of the position of the atoms, $x_{i}$ and $x_{s}$. Plots (a-d) are averaged over every unit cell to give better visibility to slower variations. A non-averaged plot is shown in Fig. 3(e) as a sample, displaying periodic variations. To focus on the $x_{i}$ functionality in plotting $W_{\text {is }}$, we fixed $x_{s}=55 a$ to outside of the nonlinear region, as the detection probability of the guided signal photon only decays slowly further away from the nonlinear region. In Figs. 3(a) and (b) we consider the case where both signal and idler are guided modes. The probability of an idler photon to be absorbed by an atom, $W_{\text {is }}$, shows a quadratic rise as a function of $x_{i}$, similar to the quadratically rising intensity of a guided mode that is pumped with a lossless and phase-matched source of nonlinear polarization [22]. The probability of the idler photon to be generated but not absorbed

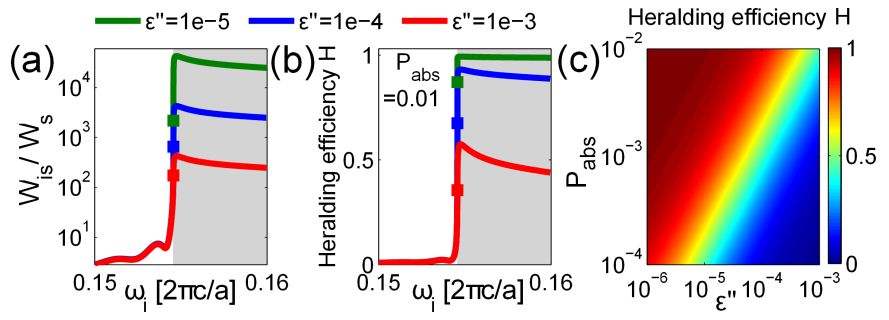

Fig. 4. (a) $W_{i s} / W_{s}$ for three different values of $\varepsilon^{\prime \prime}$. We fix $\omega_{s}=$ 0.15 and vary $\omega_{i}$. The solid squares mark where each plot passes the bandedge. The gray shaded area is the bandgap region. (b) The heralding efficiency $H$ for idler-atom's vacuum absorption probability $P_{\text {abs }}=0.01$. (c) $H$ for $\omega_{i}=0.16$ (evanescent) and $\omega_{s}=0.15$ (guided) as a function of $\varepsilon^{\prime \prime}$ and $P_{\text {abs }}$. For all plots $L=50 a, x_{s}=50 a$ and $x_{i}=25 a$.

by an atom, $W_{s}$, shows the same behavior as $W_{\text {is }}$, which means that having the photon of a guided mode absorbed by the atom or remaining in the field does not qualitatively affect the SPDC process, as $G$ and $\operatorname{Im}[G]$ are comparable for a guided mode, as shown in Fig. 2. The small periodic modulations of the graphs are due to the out-of-phase backward process. The considered losses have negligible effect on the process on this length scale.

In Figs. 3(c) and (d), the idler mode is evanescent and the signal mode is guided. $W_{\text {is }}$ increases from both ends of the nonlinear region inwards, and saturates after about twice the decay length of the evanescent mode. The saturation causes the overall SPDC efficiency to be much smaller compared to the case of both guided modes, as can be seen by comparing the numbers to Figs. 3(a) and (b). Importantly, we see that $W_{s}$ is now much smaller than $W_{\text {is }}$, due to the effect of the evanescent mode, but it is not exactly zero due to the presence of the finite loss. We see that for an order of magnitude larger $\varepsilon^{\prime \prime}, W_{s}$ also increases by an order of magnitude, clearly showing how loss can degrade our heralding mechanism. Finally, $W_{i s}$ is bound to the region inside and near $\chi^{(2)} \neq 0$, because the evanescent field can not propagate beyond the generation region. So the idler atom can only be excited near $\chi^{(2)} \neq 0$ regions, whereas the generated guided signal photon can escape the nonlinear structure.

For the final stage of our analysis, we define the heralding efficiency $H \equiv P_{i s} /\left(P_{i s}+P_{S}\right)$, which is the probability of the idler atom's excitation, in the event of a signal photon's detection. With a purely evanescent idler mode $P_{S}=0$ and $H=1$, and with loss $P_{S} \neq 0$ and $H<1$. Assuming a narrow rectangular spectrum for the pump, centered at $\omega_{s}+\omega_{i}$ with bandwidth $\Delta \omega_{p}$, over which the GF does not change considerably, we can put $\int_{0}^{\infty} d \omega_{i} \approx \Delta \omega_{i}=\Delta \omega_{p}$, where $\Delta \omega_{i}$ is the bandwidth of the generated idler photon remaining in the field. Hence we get:

$$
H=P_{\mathrm{is}} /\left(P_{\mathrm{is}}+P_{\mathrm{s}}\right) \approx 1 /\left(1+\frac{2 \pi^{2} a / \lambda_{i}}{P_{\mathrm{abs}}} \frac{W_{s}}{W_{\mathrm{is}}}\right),
$$

where $P_{\text {abs }}=2 \pi^{2}\left|d_{i}\right|^{2} /\left(\hbar \varepsilon_{0} \Delta \omega_{i} \lambda_{i} \mathcal{S}\right) \ll 1$ is the probability of the idler atom in vacuum (in 1D) absorbing a single photon wave-packet of central wavelength $\lambda_{i}$ and with a rectangular spectrum of bandwidth $\Delta \omega_{i}$, which is straightforward to calculate using the first-order perturbation theory [18].

As can be seen in Eq. (8), the $W_{\text {is }} / W_{s}$ ratio plays a key role in determining the heralding efficiency and is plotted in Fig. 4(a) for three different values of loss. Here we fixed the signal frequency to a guided mode, and varied the idler frequency. The ratio $W_{\text {is }} / W_{s}$ is much larger in the bandgap region, showing the effect 
of an evanescent idler mode in prohibiting $W_{s}$. This effect gets even stronger for smaller losses. We plot $H$ in Fig. 4(b) for a particular value of $P_{\mathrm{abs}}=0.01$. We see that $H$ is much closer to 1 in the bandgap region compared to the in-band region, and gets even closer to 1 for smaller losses. We also plot $H$ for a fixed frequency pair of a guided signal and an evanescent idler as a function of $P_{\text {abs }}$ and $\varepsilon^{\prime \prime}$ in Fig. 4(c). We see that a smaller $P_{\text {abs }}$, meaning either a shorter pump pulse in time or a weaker dipole moment of the atom, lowers $H$, but this could always be amended in a less lossy system. These results show the robustness of this heralded excitation mechanism against loss.

In order to assess the practical feasibility of emitter-mediated SPDC, we consider a PC waveguide (PCW) made of GaAs, a material with $\chi^{(2)}$ nonlinearity, which can be fabricated with a layer of quantum dots (QDs) embedded in it [10]. The bandgap region in GaAs PCWs is around $a / \lambda \approx 0.3$, hence for covering a QD's central wavelength of $\lambda_{i}=940 \mathrm{~nm}$, we need a periodicity of $a \approx 280 \mathrm{~nm}$. GaAs itself is transparent at $940 \mathrm{~nm}$. However, an equivalent $\varepsilon^{\prime \prime} \approx c \varepsilon^{\prime} /\left(n_{g} \omega L_{\text {decay }}\right)$ [25] must be assigned to the structure, where $L_{\text {decay }}$ is the scattering losses' decay length caused by fabrication imperfections. For $L_{\text {decay }} \approx 1 \mathrm{~cm}$ [26], we get $\varepsilon^{\prime \prime}\left(\omega_{i}\right) \approx 10^{-4}$. We rewrite $P_{\mathrm{abs}}=3 \lambda_{i}^{2} \Gamma_{0} /\left(4 \mathcal{S} \Delta \omega_{i} \sqrt{\varepsilon^{\prime}}\right)$, where $\Gamma_{0}=\omega_{i}^{3}|d|^{2} \sqrt{\varepsilon^{\prime}} /\left(3 \pi \hbar \varepsilon_{0} c^{3}\right)$ is the spontaneous emission rate of the emitter placed in bulk [8]. The cross-section $\mathcal{S}$ of such PCWs is roughly around $\left(\lambda_{i} / \sqrt{\varepsilon^{\prime}}\right)^{2}$, giving us $P_{\mathrm{abs}} \approx \frac{\Gamma_{0} \sqrt{\varepsilon^{\prime}}}{\Delta \omega_{i}}$. For a QD with $\Gamma_{0} / 2 \pi=0.1 \mathrm{GHz}$ [10], using a pump bandwidth of $\Delta \omega_{p} / 2 \pi=30 \mathrm{GHz}$ gives us $P_{\mathrm{abs}} \approx 0.01$, which according to Fig. 4(c) gives a heralding efficiency larger than 0.98 . To get a signal photon at the telecom wavelength of $\lambda_{s}=1550 \mathrm{~nm}$, one can use a pump central wavelength $\lambda_{p}=585 \mathrm{~nm}$. This $\lambda_{p}$ is outside the transparency window of GaAs, resulting in a very short decay length for the pump, but this could be solved using a pump-from-above scheme [27]. Considering the remarkable experimental advances made in interfacing PCs with emitters, an implementation of this proposal is within reach.

Finally, it is clear from Fig. $3(\mathrm{~d})$, where $W_{s}$ rises almost linearly with the length $L$ while $W_{\text {is }}$ saturates after a short length, that reducing $L$ should increase $W_{\text {is }} / W_{s}$ and result in $H \rightarrow 1$. However, a realistic structure is finite, causing a reflection of evanescent modes from its ends, which creates DOS at those ends that decay exponentially towards the inside of the structure [28]. Hence, $L$ has to be still large enough so that the idler atom can be placed inside the structure far enough from the ends to not get affected by this created DOS. This means an optimumlength exists in a practical design based on the amount of loss [24] to maximize the heralding efficiency.

In summary, we showed analytically that SPDC involving an evanescent idler mode is prohibited, but it can be mediated by embedding a 2-level emitter near the nonlinear structure, giving rise to a unique heralded excitation mechanism. In general, interfacing matter-based and photonic qubits is of vital importance for the implementation of quantum repeaters [29], and we believe that atom-mediated SPDC can bring about new possibilities for the engineering of such components, eventually needed for constructing a quantum network [30]. Specifically, as the 2-level emitter is only capable of accepting one quantum of excitation, this hybrid source naturally suppresses multi-photonpair generation, a constant hurdle for heralded single photon sources that creates a trade-off between the generation rate and the fidelity of the heralded state [16]. Our proposal could offer a path to lifting this fundamental trade-off. Finally, the increase in the counts of the signal photon is a sign of the presence of an idler atom near the nonlinear structure, and the close to 1 heralding efficiency essentially means a low noise sensing, offering a new scheme for nonlinear quantum spectroscopy [31].

Funding. Australian Research Council (ARC) (DP160100619); Erasmus Mundus (NANOPHI 2013 5659/002-001); Alexander von Humboldt- Stiftung; Australia-Germany Joint Research Cooperation Scheme of Universities Australia and German Academic Exchange Service; "Basis" Foundation; Russian President Grant MK-8500.2016.2; German Federal Ministry of Education and Research (FKZ 03ZZ0434, 13N14147).

\section{REFERENCES}

1. J. D. Joannopoulos, S. G. Johnson, J. N. Winn, and R. D. Meade, Photonic crystals: molding the flow of light (Princeton university press, 2011).

2. J. L. O’brien, A. Furusawa, and J. Vučković, Nat. Photonics 3, 687 (2009).

3. M. J. Collins, C. Xiong, I. H. Rey, T. D. Vo, J. He, S. Shahnia, C. Reardon, T. F. Krauss, M. J. Steel, A. S. Clark, and B. J. Eggleton, Nat. Commun. 4, 2582 (2013).

4. S. Saravi, T. Pertsch, and F. Setzpfandt, Phys. Rev. Lett. 118, 183603 (2017).

5. A. Goban, C.-L. Hung, S.-P. Yu, J. D. Hood, J. A. Muniz, J. H. Lee, M. J. Martin, A. C. McClung, K. S. Choi, D. E. Chang, O. Painter, and H. J. Kimble, Nat. Commun. 5, 3808 (2014).

6. J. S. Douglas, H. Habibian, C.-L. Hung, A. Gorshkov, H. J. Kimble, and D. E. Chang, Nat. Photonics 9, 326 (2015).

7. A. Asenjo-Garcia, J. Hood, D. Chang, and H. Kimble, Phys. Rev. A 95, 033818 (2017).

8. V. M. Rao and S. Hughes, Phys. Rev. B 75, 205437 (2007).

9. S. Noda, M. Fujita, and T. Asano, Nat. Photonics 1, 449 (2007).

10. A. Faraon, A. Majumdar, D. Englund, E. Kim, M. Bajcsy, and J. Vučković, New J. Phys. 13, 055025 (2011).

11. P. Lodahl, S. Mahmoodian, and S. Stobbe, Rev. Mod. Phys. 87, 347 (2015).

12. R. McPhedran, L. Botten, J. McOrist, A. Asatryan, C. M. de Sterke, and N. Nicorovici, Phys. Rev. E 69, 016609 (2004).

13. S. John and J. Wang, Phys. Rev. Lett. 64, 2418 (1990).

14. S. Tanzilli, A. Martin, F. Kaiser, M. P. De Micheli, O. Alibart, and D. B. Ostrowsky, Laser Photonics Rev. 6, 115 (2012).

15. J.-W. Pan, Z.-B. Chen, C.-Y. Lu, H. Weinfurter, A. Zeilinger, and M. Żukowski, Rev. Mod. Phys. 84, 777 (2012).

16. A. Christ and C. Silberhorn, Phys. Rev. A 85, 023829 (2012).

17. A. Siegman, Appl. Phys. B 60, 247 (1995).

18. W. Vogel and D.-G. Welsch, Quantum optics (John Wiley \& Sons, 2006).

19. A. N. Poddubny, I. V. Iorsh, and A. A. Sukhorukov, Phys. Rev. Lett. 117, 123901 (2016).

20. P. Y. Chen, R. C. McPhedran, C. M. de Sterke, C. G. Poulton, A. A. Asatryan, L. C. Botten, and M. J. Steel, Phys. Rev. A 82, 053825 (2010).

21. A. A. Sukhorukov, A. S. Solntsev, S. S. Kruk, D. N. Neshev, and Y. S. Kivshar, Opt. Lett. 39, 462 (2014).

22. A. Yariv and P. Yeh, Optical waves in crystals (Wiley, New York, 1984).

23. S. Saravi, S. Diziain, M. Zilk, F. Setzpfandt, and T. Pertsch, Phys. Rev. A 92, 063821 (2015).

24. S. Saravi, R. Quintero-Bermudez, F. Setzpfandt, N. A. Mortensen, and T. Pertsch, Opt. Lett. 41, 3110 (2016).

25. T. P. White and A. A. Sukhorukov, Phys. Rev. A 85, 043819 (2012).

26. S. Combrié, E. Weidner, A. DeRossi, S. Bansropun, S. Cassette, A. Talneau, and H. Benisty, Opt. Express 14, 7353 (2006).

27. A. Orieux, A. Eckstein, A. Lemaître, P. Filloux, I. Favero, G. Leo, T. Coudreau, A. Keller, P. Milman, and S. Ducci, Phys. Rev. Lett. 110, 160502 (2013).

28. E. Yeganegi, A. Lagendijk, A. P. Mosk, and W. L. Vos, Phys. Rev. B 89, 045123 (2014). 
29. F. Bussières, N. Sangouard, M. Afzelius, H. de Riedmatten, C. Simon, and W. Tittel, J. Mod. Opt. 60, 1519 (2013).

30. H. Kimble, Nature 453, 1023 (2008).

31. D. A. Kalashnikov, A. V. Paterova, S. P. Kulik, and L. A. Krivitsky, Nat. Photonics 10, 98 (2016).

\section{REFERENCES}

1. J. D. Joannopoulos, S. G. Johnson, J. N. Winn, and R. D. Meade, Photonic crystals: molding the flow of light (Princeton university press, 2011).

2. J. L. O'brien, A. Furusawa, and J. Vučković, "Photonic quantum technologies," Nat. Photonics 3, 687 (2009).

3. M. J. Collins, C. Xiong, I. H. Rey, T. D. Vo, J. He, S. Shahnia, C. Reardon, T. F. Krauss, M. J. Steel, A. S. Clark, and B. J. Eggleton, "Integrated spatial multiplexing of heralded single-photon sources," Nat. Commun. 4, 2582 (2013).

4. S. Saravi, T. Pertsch, and F. Setzpfandt, "Generation of counterpropagating path-entangled photon pairs in a single periodic waveguide," Phys. Rev. Lett. 118, 183603 (2017).

5. A. Goban, C.-L. Hung, S.-P. Yu, J. D. Hood, J. A. Muniz, J. H. Lee, M. J. Martin, A. C. McClung, K. S. Choi, D. E. Chang, O. Painter, and H. J. Kimble, "Atom-light interactions in photonic crystals," Nat. Commun. 5, 3808 (2014).

6. J. S. Douglas, H. Habibian, C.-L. Hung, A. Gorshkov, H. J. Kimble, and D. E. Chang, "Quantum many-body models with cold atoms coupled to photonic crystals," Nat. Photonics 9, 326 (2015).

7. A. Asenjo-Garcia, J. Hood, D. Chang, and H. Kimble, "Atom-light interactions in quasi-one-dimensional nanostructures: A green's-function perspective," Phys. Rev. A 95, 033818 (2017).

8. V. M. Rao and S. Hughes, "Single quantum-dot purcell factor and $\beta$ factor in a photonic crystal waveguide," Phys. Rev. B 75, 205437 (2007).

9. S. Noda, M. Fujita, and T. Asano, "Spontaneous-emission control by photonic crystals and nanocavities," Nat. Photonics 1, 449 (2007).

10. A. Faraon, A. Majumdar, D. Englund, E. Kim, M. Bajcsy, and J. Vučković, "Integrated quantum optical networks based on quantum dots and photonic crystals," New J. Phys. 13, 055025 (2011).

11. P. Lodahl, S. Mahmoodian, and S. Stobbe, "Interfacing single photons and single quantum dots with photonic nanostructures," Rev. Mod. Phys. 87, 347 (2015).

12. R. McPhedran, L. Botten, J. McOrist, A. Asatryan, C. M. de Sterke, and N. Nicorovici, "Density of states functions for photonic crystals," Phys. Rev. E 69, 016609 (2004)

13. S. John and J. Wang, "Quantum electrodynamics near a photonic band gap: Photon bound states and dressed atoms," Phys. Rev. Lett. 64 2418 (1990).

14. S. Tanzilli, A. Martin, F. Kaiser, M. P. De Micheli, O. Alibart, and D. B. Ostrowsky, "On the genesis and evolution of integrated quantum optics," Laser Photonics Rev. 6, 115 (2012).

15. J.-W. Pan, Z.-B. Chen, C.-Y. Lu, H. Weinfurter, A. Zeilinger, and M. Żukowski, "Multiphoton entanglement and interferometry," Rev. Mod. Phys. 84, 777 (2012).

16. A. Christ and C. Silberhorn, "Limits on the deterministic creation of pure single-photon states using parametric down-conversion," Phys. Rev. A 85, 023829 (2012)

17. A. Siegman, "Lasers without photons-or should it be lasers with too many photons?" Appl. Phys. B 60, 247 (1995).

18. W. Vogel and D.-G. Welsch, Quantum optics (John Wiley \& Sons, 2006).

19. A. N. Poddubny, I. V. lorsh, and A. A. Sukhorukov, "Generation of photon-plasmon quantum states in nonlinear hyperbolic metamaterials," Phys. Rev. Lett. 117, 123901 (2016).

20. P. Y. Chen, R. C. McPhedran, C. M. de Sterke, C. G. Poulton, A. A. Asatryan, L. C. Botten, and M. J. Steel, "Group velocity in lossy periodic structured media," Phys. Rev. A 82, 053825 (2010).

21. A. A. Sukhorukov, A. S. Solntsev, S. S. Kruk, D. N. Neshev, and Y. S. Kivshar, "Nonlinear coupled-mode theory for periodic plasmonic waveguides and metamaterials with loss and gain," Opt. Lett. 39, 462 (2014).

22. A. Yariv and P. Yeh, Optical waves in crystals (Wiley, New York, 1984).

23. S. Saravi, S. Diziain, M. Zilk, F. Setzpfandt, and T. Pertsch, "Phasematched second-harmonic generation in slow-light photonic crystal waveguides," Phys. Rev. A 92, 063821 (2015).

24. S. Saravi, R. Quintero-Bermudez, F. Setzpfandt, N. A. Mortensen, and T. Pertsch, "Effect of loss on slow-light-enhanced second-harmonic 
generation in periodic nanostructures," Opt. Lett. 41, 3110 (2016).

25. T. P. White and A. A. Sukhorukov, "Transition from slow and frozen to superluminal and backward light through loss or gain in dispersionengineered waveguides," Phys. Rev. A 85, 043819 (2012).

26. S. Combrié, E. Weidner, A. DeRossi, S. Bansropun, S. Cassette, A. Talneau, and H. Benisty, "Detailed analysis by fabry-perot method of slab photonic crystal line-defect waveguides and cavities in aluminiumfree material system," Opt. Express 14, 7353 (2006).

27. A. Orieux, A. Eckstein, A. Lemaître, P. Filloux, I. Favero, G. Leo, T. Coudreau, A. Keller, P. Milman, and S. Ducci, "Direct bell states generation on a iii-v semiconductor chip at room temperature," Phys. Rev. Lett. 110, 160502 (2013).

28. E. Yeganegi, A. Lagendijk, A. P. Mosk, and W. L. Vos, "Local density of optical states in the band gap of a finite one-dimensional photonic crystal," Phys. Rev. B 89, 045123 (2014).

29. F. Bussières, N. Sangouard, M. Afzelius, H. de Riedmatten, C. Simon, and W. Tittel, "Prospective applications of optical quantum memories," J. Mod. Opt. 60, 1519 (2013).

30. H. Kimble, "The quantum internet," Nature 453, 1023 (2008).

31. D. A. Kalashnikov, A. V. Paterova, S. P. Kulik, and L. A. Krivitsky, "Infrared spectroscopy with visible light," Nat. Photonics 10, 98 (2016). 